\title{
On Humanism in Longinus and Burke's Noble Thought
}

\author{
Yuanyuan Xing \\ East China University of Science and Technology, Shanghai, China \\ Email:1440997181@qq.com
}

How to cite this paper: Xing, Y.Y. (2021) On Humanism in Longinus and Burke's Noble Thought. Open Access Library Journal, 8: e7975.

https://doi.org/10.4236/oalib.1107975

Received: September 17, 2021

Accepted: November 6, 2021

Published: November 9, 2021

Copyright $\odot 2021$ by author(s) and Open Access Library Inc.

This work is licensed under the Creative Commons Attribution International License (CC BY 4.0).

http://creativecommons.org/licenses/by/4.0/

\begin{abstract}
Longinus and Burke are two important founders of the western aesthetic thought of "Sublimity". In fact, both of them contain the color of humanism when discussing this aesthetic thought. This paper attempts to analyze the humanistic color contained in Longinus and Burke's lofty thought from their source and definition of sublime.
\end{abstract}

\section{Subject Areas}

Literature, Philosophy

\section{Keywords}

Sublimity, Humanism, Longinus, Burke

\section{1. 前言}

崇高是西方美学中的一个重要思想。古希腊作家朗吉努斯在公元 1 世纪 时就首次系统地论述了崇高这一美学思想。他的著作《论崇高》探讨了对 “崇 高” 的审美感知([1]: pp.77-132)。但是这一文艺理论在一开始并没有受到太多 的重视, 直到文艺复兴时期, 才逐渐被人们发现并推崇。伯克受到了朗吉砮 斯的启发, 撰写了《关于崇高美和秀丽美概念起源的哲学探讨》 [2], 进一步 探讨了崇高这一美学思想。二者对崇高的论述既一脉相承又各自趋向不同的 侧重点。但是, 不论是在文艺复兴流行开来的《论崇高》还是在 18 世纪启蒙 时期扬名国内外的《关于崇高美和秀丽美概念起源的哲学探讨》，两者关于 崇高的论述都蕴含着人文主义色彩。本文分别从郎吉驽斯和伯克对于崇高的 定义、来源等方面来探讨其中蕴含的人文主义思想。

\section{2. 人文主义}

我们总是认为人文主义是文艺复兴时期的一种文化思潮, 其实人文主义 
是起源于古代希腊的一种文化传统, 但是不管是古希腊时期的人文主义, 还 是文艺复兴时期的人文主义以及之后各个时期的人文主义, 其思想内核总体 上都是一致的, 那就是以人为本, 追求个人价值和个人幸福, 宣扬个性解放, 追求个人反对中世纪的宗教神权。文艺复兴时期的人文主义把人从上帝那里 解放了出来, 其精神特质是世俗的、感性的。启蒙运动时期人文主义在强调 人以人为本的内核之外还散发着理性主义的光辉。总而言之, 人文主义在西 方社会中是一个永恒的话题[3]。

\section{3. 郎吉姱斯崇高思想中的人文主义}

郎吉娺斯提出了 “崇高” 这一概念, 他的《论崇高》是现存最早的论述 崇高的作品。“崇高(hypsos)”一词的内涵在郎吉驽斯之前弥漫着宗教神学色 彩。在《约翰福音》里面说到: 崇高将我们引向上帝的伟大精神。郎吉㛎斯 在崇高论述方面的贡献就是把崇高由神性转向了人性。在柏拉图强调人的思 想的基础上, 进行了更深一层的探讨, 强调人的思想高度和感情强度。

郎吉驽斯认为崇高是一种与众不同的、绝妙的表达。好的语言用其优秀 的表达方式来征服读者, 崇高的力量不可阻挡。这种崇高的表达需要拥有崇 高思想的作家来完成。他给崇高的定义注入了世俗的力量, 崇高的作品是需 要作家这个人用他崇高的思想来完成的, 而不是上帝的力量。从广义上看, 郎吉㛎斯认为, 崇高就是一切壮丽、恢弘的客体。他说: 我们并不去赞美小 溪尽管他们实用且清澈, 我们欣赏尼罗河、多瑙河和莱茵河, 我们更欣赏海 洋。崇高就是给我们留下惊心动魄的事物。这里虽然描写的是崇高的事物, 但确是按照人的感官, 人的感受来衡量什么是崇高, 在无形中强调了人的情 感、人的价值。从人的角度来认识崇高, 这一定程度上体现了其中的人文主 义思想。

另外郎吉姱斯认为崇高有五个方面的来源: 第一、形成伟大思想的力量, 第二、强烈的启发性的情感, 第三、艺术技巧, 第四、高雅的措辞, 第五、 整个文章的结构很好地融合成一体。在这五个来源中最重要的是前两点。前 两点是与作家本人息息相关的, 作家需要拥有高尚的人格, 才能写出高尚的 作品, 有别于苏格拉底所说的: 创作是上帝的神谕带来的。崇高性兼具思想 性和情感的力量, 郎吉㛎斯说: “崇高是伟大心灵的回声。” “伟大的心灵 包括两个方面: 一是思想, 一是情感, 思想要庄严伟大, 情感要激昂强烈, 前者是理性的光辉, 后者是感性的丰彩, 前者高瞻远瞩, 仰视真理, 包涵智 慧和美德, 后者心荡神驰, 激发灵感, 具备磁性和魔力。” ([4]: p.54)崇高源 于主体的心灵, 具有主观精神性。

总之, 郎吉㛎斯的《论崇高》在文艺复兴时期能够流行开来, 不仅仅是 因为这篇文论中独特的美学思想, 评论方法, 还因为其中处处渗透着符合当 时时代潮流的人文主义思想。

\section{4. 伯克崇高思想中的人文主义}

埃德蒙 - 伯克的《关于崇高美和秀丽美起源的哲学探讨》不仅是关于崇 高的主要理论之一, 也是现代美学的奠基之作。伯克的美学观念是建立在人 
的感官感觉之上的, 对新事物的好奇同时也引起我们的快感和痛感, 由痛感 和快感引申出了崇高美和秀丽美。

伯克对崇高的定义不同于郎吉驽斯对崇高的定义, 也不同于我们通常认 为的崇高。伯克在论述崇高时, 以作家本人为论述对象, 来探讨在文学创作 中如何获得崇高美。伯克认为崇高就是任何可以引起人们心中强烈感情的事 物, 具体来说是引起人心中恐惧、痛苦等情感的事物。因为在伯克看来, 痛 苦的情感比快乐的情感更强烈。伯克的这种对情感强度的讨论为建立一种关 于人和社会的更普遍的哲学人类学的观点奠定了基础。

在伯克的论述中，崇高美和秀丽美是相互交织的，两者常常交互定义， 因此孤立的谈任何一个都是没有意义的。伯克把崇高美和秀丽美进行比较, 认为崇高就是粗粘的、模糊的, 宏大的。这些对崇高的表述都是从人的感觉 来进行表述的, 这就肯定了人的地位。无论是从伯克关于崇高的论述对象方 面, 还是从他对崇高的来源表述中强调人的感觉方面, 都可以看出伯克的崇 高思想蕴含着丰富的人文主义色彩。在强调人的价值、人的作用方面伯克与 郎吉㛎斯有相通之处, 但是伯克在论述崇高中还闪炼着理性主义的光辉, 他 强调我们的感觉需要以正确的价值判断为前提。崇高美和秀丽美还分别从属 不同的领域，崇高美属于个人主义的范畴，秀丽美属于社会政治范畴。伯克 认为: 如果人类所能感受到的最强烈的情感是面对死亡时的恐惧, 那么自我 保护的本能就是人类所能拥有的最强烈体验的来源, 因此与自我保护关系不 大的社会情感的作用就相对较弱 [5]。通过强调与社会不同的个人力量, 伯克 为与崇高相关联的英雄主义主体性奠定了基础。

伯克还认为崇高具有超越性。崇高是审美主体的审美理性能力超越审美 客体的表现。他从郎吉驽斯的 “被真正的崇高升华是我们的天性。充满喜悦 和骄傲, 开始相信我们所创造的只是我们听到的” 中受到启发, 将恐惧与自 我的尊严和庄严联系在一起, 也就是说, “膨胀” 不仅仅是自我主义, 而是 涉及灵魂的崇高。“胜利”、“荣耀”、“内在的伟大” 等词将英雄价值与 资产阶级雄心(向上流动)的崇高联系起来。一个人被恐怖抬高了, 也就是说, 崇高允许我们利用激发我们恐惧的力量 [6]。

\section{5. 结论}

郎吉驽斯和伯克都把崇高联系于主体人性，郎吉驽斯的崇高论述除强调 了人的感觉、感情之外, 体现了人文主义忽视上帝的作用, 强调人的价值, 人的作用。他把崇高从原来的 “神性” 逐渐转变为 “人性”。坚持以人为本, 把个人作为崇高讨论的中心。伯克在强调人的感官感觉的基础上，对于崇高 的论述更具有理性色彩。这使得崇高中的人文主义得到了一定的发展, 理性 主义成为主旋律，但是其思想内核也是在强调理性的基础上，重视人的价值、 人的作用。

\section{Conflicts of Interest}

The author declares no conflicts of interest. 


\section{References}

[1] 朗吉努斯. 论崇高 $[M] / /$ 章安祺, 编. 缪灵珠美学译文集(卷 1). 北京: 中国人民大 学出版社, 1998.

[2] 伯克. 关于我们崇高与美观念之根源的哲学探讨 $[M]$. 郭飞, 译. 郑州: 大象出版 社, 2010 .

[3] 黄伊梅. 希腊古典人文主义的内涵与特质[J]. 学术研究, 2008(12): 38-43.

[4] 李思孝. 郎吉㛎斯《论崇高》的美学价值[J]. 求实学刊, 1989(4): 50-56.

[5] 彭立勋. 伯克论崇高与美[J]. 武汉理工大学学报(社会科学版), 2004, 17(3): 289-295.

[6] 陈榕. 恐怖及其观众: 伯克崇高论中的情感、政治与伦理[J]. 外国文学, 2020(6): 130-143.

\section{Appendix (Abstract and Keywords in Chinese) 浅析朗吉驽斯和伯克崇高思想中的人文主义}

摘要：郎吉㛎斯和伯克是西方 “崇高” 这一美学思想的两位重要的奠基人, 他们两者在论述这一美学思想的时候其实都包含着人文主义色彩。本文试图 从他们对于崇高的来源、定义等方面来分析郎吉驽斯和伯克的崇高思想中所 蕴含的人文主义色彩。

关键词: 崇高, 人文主义, 郎吉驽斯, 伯克 